\title{
Generalized bent Boolean functions and strongly regular Cayley graphs
}

\author{
Constanza Riera ${ }^{1}$, Pantelimon Stănică ${ }^{2}$, Sugata Gangopadhyay ${ }^{3}$, \\ ${ }^{1}$ Department of Computing, Mathematics, and Physics, \\ Western Norway University of Applied Sciences, \\ 5020 Bergen, Norway; csr@hvl.no \\ ${ }^{2}$ Department of Applied Mathematics, \\ Naval Postgraduate School, \\ Monterey, CA 93943, USA; pstanica@nps.edu \\ ${ }^{3}$ Department of Computer Science and Engineering \\ Indian Institute of Technology Roorkee, \\ Roorkee 247667, INDIA; gsugata@gmail.com
}

November 21, 2017

\begin{abstract}
In this paper we define the (edge-weighted) Cayley graph associated to a generalized Boolean function, introduce a notion of strong regularity and give several of its properties. We show some connections between this concept and generalized bent functions (gbent), that is, functions with flat Walsh-Hadamard spectrum. In particular, we find a complete characterization of quartic gbent functions in terms of the strong regularity of their associated Cayley graph.
\end{abstract}

\section{1 (Generalized) Boolean functions background}

Let $\mathbb{V}_{n}$ be the vector space of dimension $n$ over the two element field $\mathbb{F}_{2}$, and for a positive integer $q$, let $\mathbb{Z}_{q}$ be the ring of integers modulo $q$. Let us denote the addition, respectively, product operators over $\mathbb{F}_{2}$ by " $\oplus$ ", respectively, ".". A Boolean function $f$ on $n$ variables is a mapping from $\mathbb{V}_{n}$ into $\mathbb{F}_{2}$, that is, a multivariate polynomial over $\mathbb{F}_{2}$,

$$
f\left(x_{1}, \ldots, x_{n}\right)=a_{0} \oplus \sum_{i=1}^{n} a_{i} x_{i} \oplus \sum_{1 \leq i<j \leq n} a_{i j} x_{i} x_{j} \oplus \ldots \oplus a_{12 \ldots n} x_{1} x_{2} \ldots x_{n},
$$

where the coefficients $a_{0}, a_{i}, a_{i j}, \ldots, a_{12 \ldots n} \in \mathbb{F}_{2}$. This representation of $f$ is called the algebraic normal form (ANF) of $f$. The number of variables in the highest order product term with nonzero coefficient is called the algebraic degree, or simply the degree of $f$.

For a Boolean function on $\mathbb{V}_{n}$, the Hamming weight of $f$, wt $(f)$, is the cardinality of $\Omega_{f}=\left\{\mathbf{x} \in \mathbb{V}_{n}: f(\mathbf{x})=1\right\}$ (this is extended to any vector, by taking its weight to 
be the number of nonzero components of that vector). The Hamming distance between two functions $f, g: \mathbb{V}_{n} \rightarrow \mathbb{F}_{2}$ is $d(f, g)=w t(f \oplus g)$. A Boolean function $f(\mathbf{x})$ is called an affine function if its algebraic degree is 1 . If, in addition, $a_{0}=0$ in (1), then $f(\mathbf{x})$ is a linear function (see [8] for more on Boolean functions). In $\mathbb{V}_{n}=\mathbb{F}_{2}^{n}$, the vector space of the $n$-tuples over $\mathbb{F}_{2}$, we use the conventional dot product $\mathbf{u} \cdot \mathbf{x}$ as an inner product.

For a generalized Boolean function $f: \mathbb{V}_{n} \rightarrow \mathbb{Z}_{q}$ we define the generalized WalshHadamard transform to be the complex valued function

$$
\mathcal{H}_{f}^{(q)}(\mathbf{u})=\sum_{\mathbf{x} \in \mathbb{V}_{n}} \zeta_{q}^{f(\mathbf{x})}(-1)^{\mathbf{u} \cdot \mathbf{x}}
$$

where $\zeta_{q}=e^{\frac{2 \pi i}{q}}$ (we often use $\zeta, \mathcal{H}_{f}$, instead of $\zeta_{q}$, respectively, $\mathcal{H}_{f}^{(q)}$, when $q$ is fixed). The inverse is given by $\zeta^{f(\mathbf{x})}=2^{-n} \sum_{\mathbf{u}} \mathcal{H}_{f}(\mathbf{u})(-1)^{\mathbf{u} \cdot \mathbf{x}}$. For $q=2$, we obtain the usual Walsh-Hadamard transform

$$
\mathcal{W}_{f}(\mathbf{u})=\sum_{\mathbf{x} \in \mathbb{V}_{n}}(-1)^{f(\mathbf{x})}(-1)^{\mathbf{u} \cdot \mathbf{x}}
$$

which defines the coefficients of character form of $f$ with respect to the orthonormal basis of the group characters $\chi_{\mathbf{w}}(\mathbf{x})=(-1)^{\mathbf{w} \cdot \mathbf{x}}$. In turn, $f(\mathbf{x})=2^{-n} \sum_{\mathbf{w}} \mathcal{W}_{f}(\mathbf{w})(-1)^{\mathbf{u} \cdot \mathbf{x}}$.

We use the notation as in [10, 11, 12, 15, 16] (see also [14, 17]) and denote the set of all generalized Boolean functions by $\mathcal{G B}_{n}^{q}$ and when $q=2$, by $\mathcal{B}_{n}$. A function $f: \mathbb{V}_{n} \rightarrow \mathbb{Z}_{q}$ is called generalized bent (gbent) if $\left|\mathcal{H}_{f}(\mathbf{u})\right|=2^{n / 2}$ for all $\mathbf{u} \in \mathbb{V}_{n}$. We recall that a function $f$ for which $\left|\mathcal{W}_{f}(\mathbf{u})\right|=2^{n / 2}$ for all $\mathbf{u} \in \mathbb{V}_{n}$ is called a bent function, which only exist for even $n$ since $\mathcal{W}_{f}(\mathbf{u})$ is an integer. Let $f \in \mathcal{G B}_{n}^{q}$, where $2^{k-1}<q \leq 2^{k}$, then we can represent $f$ uniquely as

$$
f(\mathbf{x})=a_{0}(\mathbf{x})+2 a_{1}(\mathbf{x})+\cdots+2^{k-1} a_{k-1}(\mathbf{x})
$$

for some Boolean functions $a_{i}, 0 \leq i \leq k-1$ (this representation comes from the binary representation of the elements in the image set $\mathbb{Z}_{2^{k}}$ ). For results on classical bent functions and related topics, the reader can consult [5, 8, 13, 18].

\section{Unweighted strongly regular graphs}

A graph is regular of degree $r$ (or $r$-regular) if every vertex has degree $r$ (number of edges incident to it). We say that an $r$-regular graph $G$ is a strongly regular graph ( $\mathrm{srg}$ ) with parameters $(v, r, e, d)$ if there exist nonnegative integers $e, d$ such that for all vertices $\mathbf{u}, \mathbf{v}$ the number of vertices adjacent to both $\mathbf{u}, \mathbf{v}$ is $d, e$, if $\mathbf{u}, \mathbf{v}$ are adjacent, respectively, nonadjacent (see for instance [9]). The complementary graph $\bar{G}$ of the strongly regular graph $G$ is also strongly regular with parameters $(v, v-r-1, v-2 r+e-2, v-2 r+d)$ (see [9]).

Since the objects of this paper are edge-weighted graphs $G=(V, E, w)$ (with vertices $V$, edges $E$ and weight function $w$ defined on $E$ with values in some set, which in our case it will be either the set of integers modulo $q, \mathbb{Z}_{q}$ with $q=2^{k}$, or the complex 
numbers set $\mathbb{C}$ ), we define the weighted degree $d(v)$ of a vertex $v$ to be the sum of the weights of its incident edges, that is, $d(v)=\sum_{u,(u, v) \in E} w(u, v)$ (later, we will introduce yet another degree or strength concept). Certainly, one can also define the combinatorial degree $r(v)$ of a vertex to be the number of such incident edges. For more on graph theory the reader can consult [4, 9] or one's favorite graph theory book.

Let $f$ be a Boolean function on $\mathbb{V}_{n}$. We define the Cayley graph of $f$ to be the graph $G_{f}=\left(\mathbb{V}_{n}, E_{f}\right)$ whose vertex set is $\mathbb{V}_{n}$ and the set of edges is defined by

$$
E_{f}=\left\{(\mathbf{w}, \mathbf{u}) \in \mathbb{V}_{n} \times \mathbb{V}_{n}: f(\mathbf{w} \oplus \mathbf{u})=1\right\} .
$$

For some fixed (but understood from the context) positive integer $s$, let the canonical injection $\iota: \mathbb{V}_{s} \rightarrow \mathbb{Z}_{2^{s}}$ be defined by $\iota(\mathbf{c})=\mathbf{c} \cdot\left(1,2, \ldots, 2^{s-1}\right)=\sum_{j=0}^{s-1} c_{j} 2^{j}$, where $\mathbf{c}=\left(c_{0}, c_{1}, \ldots, c_{s-1}\right)$. For easy writing, we denote by $\mathbf{j}:=\iota^{-1}(j)$.

The adjacency matrix $A_{f}$ is the matrix whose entries are $A_{i, j}=f(\mathbf{i} \oplus \mathbf{j}$ ) (here $\iota$ is defined on $\left.\mathbb{V}_{n}\right)$. It is simple to prove that $A_{f}$ has the dyadic property: $A_{i, j}=$ $A_{i+2^{k-1}, j+2^{k-1}}$. Also, from its definition, we derive that $G_{f}$ is a regular graph of degree $w t(f)=\left|\Omega_{f}\right|$ (see [9, Chapter 3] for further definitions).

Given a graph $f$ and its adjacency matrix $A$, the spectrum, with notation $\operatorname{Spec}\left(G_{f}\right)$, is the set of eigenvalues of $A$ (called also the eigenvalues of $G_{f}$ ). We assume throughout that $G_{f}$ is connected (in fact, one can show that all connected components of $G_{f}$ are isomorphic).

It is known (see [9, pp. 194-195]) that a connected $r$-regular graph is strongly regular iff it has exactly three distinct eigenvalues $\lambda_{0}=r, \lambda_{1}, \lambda_{2}$ (so $e=r+\lambda_{1} \lambda_{2}+\lambda_{1}+\lambda_{2}$, $\left.d=r+\lambda_{1} \lambda_{2}\right)$.

The following result is known [9, Th. 3.32, p. 103] (the second part follows from a counting argument and is also well known).

Proposition 1. The following identity holds for a strongly r-regular graph:

$$
A^{2}=(d-e) A+(r-e) I+e J
$$

where $J$ is the all 1 matrix. Moreover, $r(r-d-1)=e(v-r-1)$.

In [1, 2] it was shown that a Boolean function $f$ is bent if and only if the Cayley graph $G_{f}$ is strongly regular with $e=d$. We shall refer to this as the BernasconiCodenotti correspondence.

\section{The Cayley graph of a generalized Boolean function}

We now let $f: \mathbb{V}_{n} \rightarrow \mathbb{Z}_{q}$ be a generalized Boolean function. We define the (generalized) Cayley graph $G_{f}$ to be the graph where vertices are the elements of $\mathbb{V}_{n}$ and two vertices $\mathbf{u}, \mathbf{v}$ are connected by a weighted edge of (multiplicative) weight $\zeta^{f(\mathbf{u} \oplus \mathbf{v})}$ (respectively, additive weight $f(\mathbf{u} \oplus \mathbf{v}))$. Certainly, the underlying unweighted graph is a complete pseudograph (every vertex also has a loop). We sketch in Figure 1 such an example. 


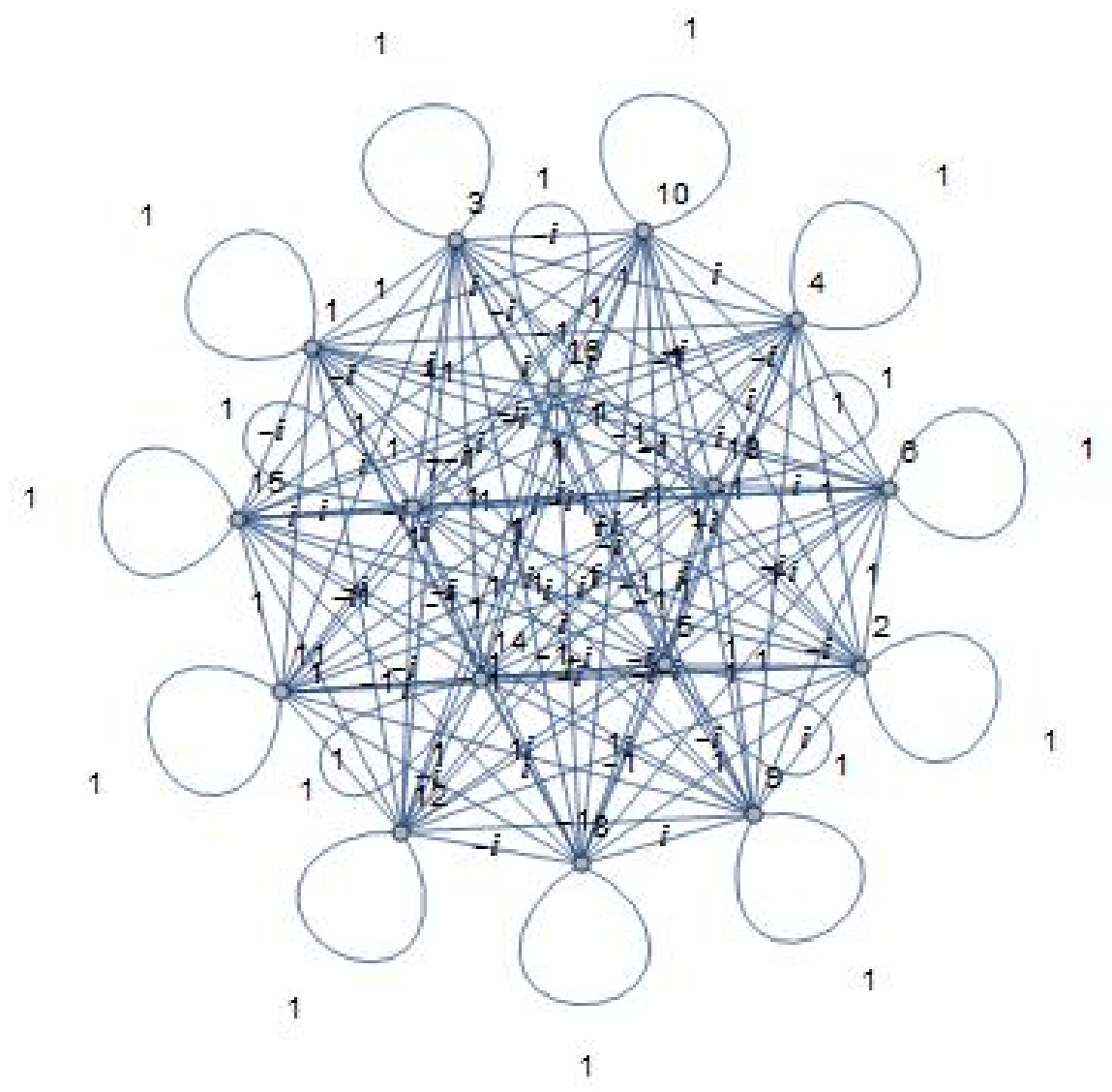

Figure 1: Cayley graph associated to the gbent $f(\mathbf{x})=x_{1}+2\left(x_{1} x_{2} \oplus x_{3} x_{4}\right)$

Certainly, one can define a modified (generalized) Cayley graph $G_{f}^{\prime}$ where two vertices are connected if and only if $f(\mathbf{u} \oplus \mathbf{v}) \neq 0$ with weights given by $\zeta^{f(\mathbf{u} \oplus \mathbf{v})}$. We sketch in Figure 2 such a graph (it is ultimately the above graph with all weight 1 edges removed).

In Example 2, we give an example of a generalized Cayley graph, and its spectrum.

Example 2. Let $f: \mathbb{V}_{n} \rightarrow \mathbb{Z}_{4}$ defined by $f\left(x_{1}, x_{2}\right)=x_{1} x_{2}+2 x_{1}$. The truth table is $\left(\begin{array}{llll}0 & 0 & 2 & 3\end{array}\right)^{T}$ (using the lexicographical order $\left.x_{1}, x_{2}\right)$. Then, the adjacency matrix (with multiplicative weights) is

$$
A_{f}=\left(\begin{array}{cccc}
1 & 1 & -1 & -i \\
1 & 1 & -i & -1 \\
-1 & -i & 1 & 1 \\
-i & -1 & 1 & 1
\end{array}\right)
$$

$A$ basis for its eigenspace is $\left\{\vec{v}_{1}, \vec{v}_{2}, \vec{v}_{3}, \vec{v}_{4}\right\}$, where $\vec{v}_{1}=\left(\begin{array}{llll}1 & 1 & 1 & 1\end{array}\right)^{T}$ with $\chi_{1}(\mathbf{x})=(-1)^{0}$ $\vec{v}_{2}=\left(\begin{array}{llll}1 & -1 & 1 & -1\end{array}\right)^{T}$ with $\chi_{2}(\mathbf{x})=(-1)^{x_{2}}, \vec{v}_{3}=\left(\begin{array}{llll}1 & 1 & -1 & -1\end{array}\right)^{T}$ with $\chi_{3}(\mathbf{x})=(-1)^{x_{1}}$, $\vec{v}_{4}=\left(\begin{array}{llll}1 & -1 & -1 & 1\end{array}\right)^{T}$ with $\chi_{4}(\mathbf{x})=(-1)^{x_{1}+x_{2}}$, having respective eigenvalues $\lambda_{0}=$ 


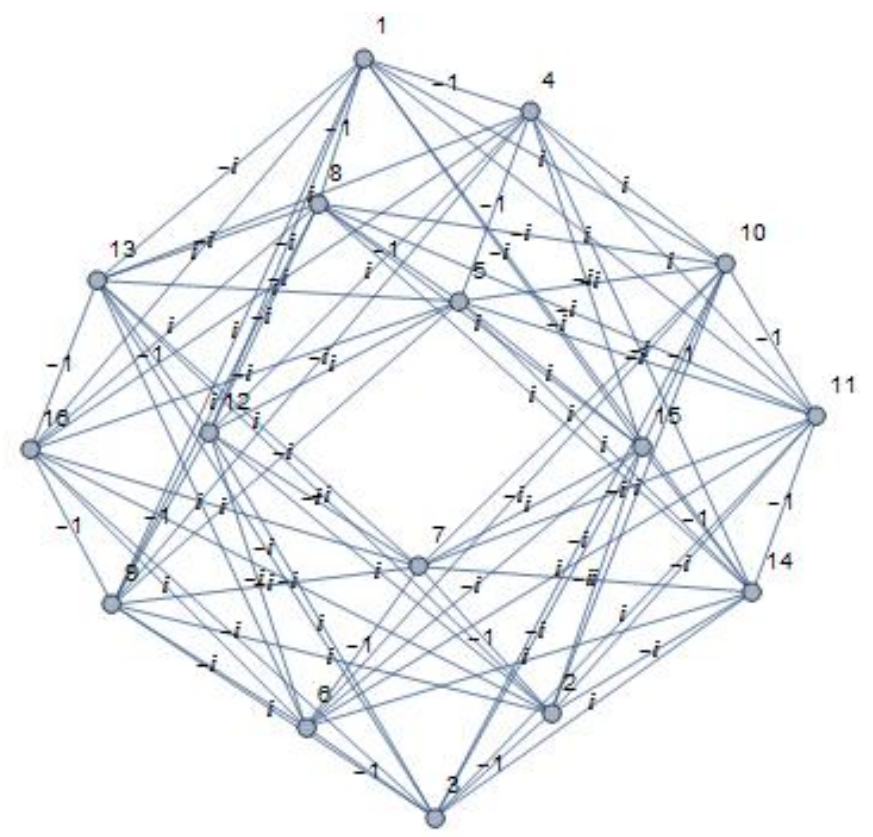

Figure 2: Modified Cayley graph associated to gbent $f(\mathbf{x})=x_{1}+2\left(x_{1} x_{2} \oplus x_{3} x_{4}\right)$

$1-i, \lambda_{1}=-1+i, \lambda_{2}=3+i, \lambda_{3}=1-i$. We can see that the eigenvalues $A_{f}$ are

$$
\begin{aligned}
& \lambda_{0}=i^{0} \chi_{1}(00)+i^{0} \chi_{1}(01)+i^{2} \chi_{1}(10)+i^{3} \chi_{1}(11)=1+1+i^{2}+i^{3}=1-i=\mathcal{H}_{f}^{(4)}(\mathbf{0}), \\
& \lambda_{1}=i^{0} \chi_{2}(00)+i^{0} \chi_{2}(01)+i^{2} \chi_{2}(10)+i^{3} \chi_{2}(11)=1-1+i^{2}-i^{3}=-1+i=\mathcal{H}_{f}^{(4)}(\mathbf{1}), \\
& \lambda_{2}=i^{0} \chi_{3}(00)+i^{0} \chi_{3}(01)+i^{2} \chi_{3}(10)+i^{3} \chi_{3}(11)=1+1-i^{2}-i^{3}=3+i=\mathcal{H}_{f}^{(4)}(\mathbf{2}), \\
& \lambda_{3}=i^{0} \chi_{4}(00)+i^{0} \chi_{4}(01)+i^{2} \chi_{4}(10)+i^{3} \chi_{4}(11)=1-1-i^{2}+i^{3}=1-i=\mathcal{H}_{f}^{(4)}(\mathbf{3}) .
\end{aligned}
$$

Although, we do not use it in this paper, we define the strength of the vertex a in the Cayley graph $G_{f}$ as the sum of the additive weights of incident edges, that is, $s(\mathbf{a})=\sum_{b} f(\mathbf{a} \oplus \mathbf{b})$.

Remark 3. If $f \in \mathcal{G B}_{n}^{q}$ and $G_{f}$ is its Cayley graph, we observe that all vertices are adjacent of multiplicative (respectively, additive) weights in $\mathbb{U}_{q}=\left\{1, \zeta, \zeta^{2}, \ldots, \zeta^{q-1}\right\}$ (respectively, in $\mathbb{Z}_{q}=\{0,1 \ldots, q-1\}$ ).

We next show that the eigenvalues of the Cayley graph $G_{f}$ (with multiplicative weights) are precisely the (generalized) Walsh-Hadamard coefficients.

Theorem 4. Let $f: \mathbb{V}_{n} \rightarrow \mathbb{Z}_{q}, q=2^{k}$, and let $\lambda_{i}, 0 \leq i \leq 2^{n}-1$ be the eigenvalues of its associated (multiplicative) edge-weighted graph $G_{f}$. Then,

$$
\lambda_{i}=\mathcal{H}_{f}^{(q)}(\mathbf{i})\left(\text { recall that } \mathbf{i}=\iota^{-1}(i)\right) .
$$

Proof. Let $\chi: \mathbb{V}_{n} \rightarrow \mathbb{C}$ be a character of $\mathbb{V}_{n}$, and for each such character, let $\mathbf{x}_{\chi}=$ $\left(x_{j}\right)_{0 \leq j \leq 2^{n}-1} \in \mathbb{C}^{2^{n}}$, where $x_{j}=\chi(\mathbf{j})$. We claim (and show) that $\mathbf{x}_{\chi}$ is an eigenvector 
of $A=A_{f}$ (for simplicity, we use $A$ in lieu of $A_{f}$ in this proof), with eigenvalue $\sum_{k=0}^{q-1} \sum_{\mathbf{s}_{\mathbf{k}} \in S_{k}} \zeta^{k} \chi\left(\mathbf{s}_{\mathbf{k}}\right)$, where $S_{k}=\left\{\mathbf{s}_{k}: f\left(\mathbf{s}_{k}\right)=k\right\}$. (Observe that the characters of $\mathbb{V}_{n}$ are $\chi_{\mathbf{w}}(\mathbf{x})=(-1)^{\mathbf{u} \cdot \mathbf{x}}$, and thus the eigenvalues are exactly the Walsh-Hadamard transform coefficients).

The $i$-th entry of $A \mathrm{x}$ is

$$
(A \mathbf{x})_{i}=\sum_{j} A_{i, j} x_{j}=\sum_{j} A_{i, j} \chi(\mathbf{j})=\sum_{k=0}^{q-1} \sum_{\mathbf{i} \oplus \mathbf{j} \in S_{k}} \zeta^{k} \chi(\mathbf{j})
$$

If $\mathbf{i} \oplus \mathbf{j} \in S_{k}$, then $\mathbf{i} \oplus \mathbf{j}=\mathbf{s}_{k}$, for some $\mathbf{s}_{k} \in S_{k}$, and so, $\mathbf{j}=\mathbf{i} \oplus \mathbf{s}_{k}$. Since $\chi$ is a character,

$$
\chi(\mathbf{j})=\chi\left(\mathbf{i} \oplus \mathbf{s}_{k}\right)=\chi(\mathbf{i}) \chi\left(\mathbf{s}_{k}\right)=x_{i} \chi\left(\mathbf{s}_{k}\right)
$$

Then,

$$
(A \mathbf{x})_{i}=\sum_{k=0}^{q-1} \sum_{\mathbf{s}_{k} \in S_{k}} \zeta^{k} x_{i} \chi\left(\mathbf{s}_{k}\right)=x_{i} \sum_{k=0}^{q-1} \sum_{\mathbf{s}_{k} \in S_{k}} \zeta^{k} \chi\left(\mathbf{s}_{k}\right),
$$

which shows our theorem.

\section{Generalized bents and their Cayley graphs}

We recall that a $q$-Butson Hadamard matrix [6] $(q-\mathrm{BH})$ of dimension $d$ is a $d \times d$ matrix $H$ with all entries $q$-th roots of unity such that $H H^{*}=d I_{d}$, where $H^{*}$ is the conjugate transpose of $H$. When $q=2, q$-BH matrices are called Hadamard matrices (where the entries are \pm 1$)$. Recall that the crosscorrelation function is defined by

$$
\mathcal{C}_{f, g}(\mathbf{z})=\sum_{\mathbf{x} \in \mathbb{V}_{n}} \zeta^{f(\mathbf{x})-g(\mathbf{x} \oplus \mathbf{z})},
$$

and the autocorrelation of $f \in \mathcal{G B}_{n}^{q}$ at $\mathbf{u} \in \mathbb{V}_{n}$ is $\mathcal{C}_{f, f}(\mathbf{u})$ above, which we denote by $\mathcal{C}_{f}(\mathbf{u})$.

Theorem 5. Let $f \in \mathcal{G B}_{n}^{q}$. Then $f$ is gbent if and only if the adjacency matrix $A_{f}$ of the (multiplicative) edge-weighted Cayley graph associated to $f$ is a q-Butson Hadamard matrix.

Proof. Let $A_{f}=\left(\zeta^{f(\mathbf{a}+\mathbf{b})}\right)_{\mathbf{a}, \mathbf{b}}$. Then, the $(\mathbf{a}, \mathbf{b})$-entry of $A_{f} \cdot \bar{A}_{f}$ is

$$
\left(A_{f} \cdot \bar{A}_{f}\right)_{\mathbf{a}, \mathbf{b}}=\sum_{\mathbf{c} \in \mathbb{V}_{n}} \zeta^{f(\mathbf{a} \oplus \mathbf{c})} \zeta^{\bar{f}(\mathbf{c} \oplus \mathbf{b})}=\sum_{\mathbf{c} \in \mathbb{V}_{n}} \zeta^{f(\mathbf{a} \oplus \mathbf{c})-f(\mathbf{c} \oplus \mathbf{b})}=\mathcal{C}_{f}(\mathbf{a} \oplus \mathbf{b}) .
$$

Now, recall from [15] that if $f, g \in \mathcal{G B}_{n}^{q}$, then

$$
\begin{aligned}
& \sum_{\mathbf{u} \in \mathbb{V}_{n}} \mathcal{C}_{f, g}(\mathbf{u})(-1)^{\mathbf{u} \cdot \mathbf{x}}=2^{-n} \mathcal{H}_{f}(\mathbf{x}) \overline{\mathcal{H}_{g}(\mathbf{x})}, \\
& \mathcal{C}_{f, g}(\mathbf{u})=2^{-n} \sum_{\mathbf{x} \in \mathbb{V}_{n}} \mathcal{H}_{f}(\mathbf{x}) \overline{\mathcal{H}_{g}(\mathbf{x})}(-1)^{\mathbf{u} \cdot \mathbf{x}} .
\end{aligned}
$$


Thus, equation (2) becomes

$$
\left(A_{f} \cdot \bar{A}_{f}\right)_{\mathbf{a}, \mathbf{b}}=2^{-n} \sum_{\mathbf{x} \in \mathbb{V}_{n}} \mathcal{H}_{f}(\mathbf{x}) \overline{\mathcal{H}_{f}(\mathbf{x})}(-1)^{(\mathbf{a} \oplus \mathbf{b}) \cdot \mathbf{x}}=2^{-n} \sum_{\mathbf{x} \in \mathbb{V}_{n}}\left\|\mathcal{H}_{f}(\mathbf{x})\right\|^{2}(-1)^{(\mathbf{a} \oplus \mathbf{b}) \cdot \mathbf{x}} .
$$

By Parseval's identity, if $\mathbf{a}=\mathbf{b}$, then $\left(A_{f} \cdot \bar{A}_{f}\right)_{\mathbf{a}, \mathbf{a}}=2^{n}$. Assume now that $\mathbf{a} \neq \mathbf{b}$ and we shall show that $\left(A_{f} \cdot \bar{A}_{f}\right)_{\mathbf{a}, \mathbf{b}}=0$ for some $\mathbf{a} \neq \mathbf{b}$ if and only if $f$ is gbent. Certainly, if $f$ is gbent then $\left\|\mathcal{H}_{f}(\mathbf{x})\right\|^{2}=2^{n}$, and since $\sum_{\mathbf{x} \in \mathbb{V}_{n}}(-1)^{(\mathbf{a} \oplus \mathbf{b}) \cdot \mathbf{x}}=0$, for $\mathbf{a} \oplus \mathbf{b} \neq 0$, we have that implication. We can certainly show it directly, but the converse follows from [15, Theorem $1(i v)]$.

For the remaining of the paper, for simplicity, we shall only consider additive weights, namely, our edge-weighted graphs $(V, E, w)$ will have the weight function $w$ : $E \rightarrow \mathbb{Z}_{q}, q=2^{k}$

Next, we say that a weighted graph $G=(V, E, w), V \subseteq \mathbb{V}_{n}, w: E \rightarrow \mathbb{Z}_{q}, q=2^{k}$, is a weighted regular graph (wrg) of parameters $\left(v ; r_{0}, r_{1}, \ldots, r_{q-1}\right)$ if every vertex will have exactly $r_{j}$ neighbors of edge weight $j$. We denote by $N_{j}(\mathbf{a})$ the set of all neighbors of a vertex a of corresponding edge weight $j$.

Proposition 6. Given a generalized Boolean function $f \in \mathcal{G B}_{n}^{q}$, the associated Cayley graph is weighted regular (of some parameters), that is, every vertex will have the same number of incident edges with a fixed weight.

Proof. Fix a weight $j$ and a vertex $\mathbf{x}_{0}$, and consider the equation $f\left(\mathbf{x}_{0} \oplus \mathbf{y}\right)=j$ with solutions $\mathbf{y}_{1}, \mathbf{y}_{2}, \ldots, \mathbf{y}_{t}$, say. For any other vertex $\mathbf{x}_{1}$, the equation $f\left(\mathbf{x}_{1} \oplus \mathbf{y}\right)=j$ will have solutions $\mathbf{y}_{1} \oplus \mathbf{x}_{1} \oplus \mathbf{x}_{0}, \mathbf{y}_{2} \oplus \mathbf{x}_{1} \oplus \mathbf{x}_{0}, \ldots, \mathbf{y}_{t} \oplus \mathbf{x}_{1} \oplus \mathbf{x}_{0}$. The proof of the lemma is done.

We will define our first concept of strong regularity here. Let $X, \bar{X}$ be a fixed bisection of the weights $\mathbb{Z}_{q}=X \cup \bar{X}, X \cap \bar{X}=\emptyset,|X|=|\bar{X}|=2^{k-1}$, and let $Y \subseteq \mathbb{Z}_{q}$. We say that a weighted regular (of parameters $\left(v ; r_{0}, r_{1}, \ldots, r_{q-1}\right)$ ) graph $G=(V, E, w)$, $V \subseteq \mathbb{V}_{n}, w: E \rightarrow \mathbb{Z}_{q}, q=2^{k}$, is a (generalized) $(X ; Y)$-strongly regular (srg) of parameters $\left(v ; r_{0}, r_{1}, \ldots, r_{q-1} ; e_{X}, d_{X}\right)$ if and only if the number of vertices $\mathbf{c}$ adjacent to both $\mathbf{a}, \mathbf{b}$, with $w(\mathbf{a}, \mathbf{c}) \in Y, w(\mathbf{b}, \mathbf{c}) \in Y$, is exactly $e_{X}$ if $w(\mathbf{a}, \mathbf{b}) \in X$, respectively, $d_{X}$ if $w(\mathbf{a}, \mathbf{b}) \in \bar{X}$. One can weaken the condition and define a $\left(X_{1}, X_{2} ; Y\right)$-srg notion, where $X_{1} \cap X_{2}=\emptyset$, not necessarily a bisection, and require the number of vertices c adjacent to both $\mathbf{a}, \mathbf{b}$, with $w(\mathbf{a}, \mathbf{c}) \in Y, w(\mathbf{b}, \mathbf{c}) \in Y$, to be exactly $e_{X}$ if $w(\mathbf{a}, \mathbf{b}) \in X_{1}$, respectively, $d_{X}$ if $w(\mathbf{a}, \mathbf{b}) \in X_{2}$; or even allowing a multi-section, and all of these variations can be fresh areas of research for graph theory experts.

Note that our definition (see also [7] for an alternative concept, which we mention in the last section) is a natural extension of the classical definition: Let $q=2$, and $X=\{1\}$. A classical strongly regular graph is then equivalent to an $(X ; X)$-strongly regular graph.

We first show that (part of) Proposition 1 can be adapted to this notion, as well, in some cases, and we deal below with one such instance. 
Proposition 7. Let $G=(V, E, w)$ be a weighted $(X ; X)$-strongly regular graph of parameters $\left(v ; r_{0}, r_{1}, \ldots, r_{q-1} ; e_{X}, d_{X}\right)$, where $X \subseteq \mathbb{Z}_{q}, v=|V|$. Then,

$$
r_{X}\left(r_{X}-e_{X}-1\right)=d_{X}\left(v-r_{X}-1\right)
$$

where $r_{X}=\sum_{i \in X} r_{i}$.

Proof. Without loss of generality we assume that the weights are additive, that is, they belong to $\mathbb{Z}_{q}$. Fix a vertex $\mathbf{u} \in V$ and let $A$ be the set of vertices adjacent to $\mathbf{u}$ with connecting edges of weight in $X$, and $B=V \backslash\{A, \mathbf{u}\}$. Observe that $|A|=\sum_{i \in X} r_{i}=r_{X}$ and $|B|=v-r_{X}-1$. We somewhat follow the combinatorial method of the classical case, and we shall count the number of vertices between $A$ and $B$ in two different ways. For any vertex $\mathbf{a} \in A$, there are exactly $e_{X}$ vertices in $A$ adjacent to both $\mathbf{u}$, a of edge weights in $X$, and so, exactly $r_{X}-e_{X}-1$ neighbors in $B$ whose connecting edges have weight in $X$. Therefore, the number of edges of weight in $X$ between $A$ and $B$ is $r_{X}\left(r_{X}-e_{X}-1\right)$.

On the other hand, any vertex $\mathbf{b} \in B$ is adjacent to $d_{X}$ vertices in $A$ of connecting edge with weight in $X$ (since $\mathbf{u}, \mathbf{b}$ must share $d_{X}$ common vertices of connecting edges of weight in $X$ ) and so, the total number of edges of weight in $X$ between $A$ and $B$ is $d_{X}\left(v-r_{X}-1\right)$. The proposition follows.

Let $G=(V, E, w)\left(w: E \rightarrow \mathbb{Z}_{q}\right)$ be a weighted graph, where $w(E) \subseteq \mathbb{Z}_{q}$ (or $\left.w(E) \subseteq \mathbb{U}_{q}\right)$. We define the complement of $G$, denoted by $\bar{G}$ the graph of vertex set $V$ with an edge between two vertices $\mathbf{a}, \mathbf{b}$ having weight $q-1-f(\mathbf{a} \oplus \mathbf{b})$ (or, multiplicatively, $\zeta^{q-1-f(\mathbf{a} \oplus \mathbf{b})}$. This is a natural definition, since if $G$ is the Cayley graph associated to $f=a_{0}+2 a_{1}, a_{0}, a_{i} \in \mathcal{B}_{n}$, then we observe that $\bar{G}$ is the Cayley graph associated to $\bar{f}=\bar{a}_{0}+2 \bar{a}_{1}+\cdots+2^{k-1} \bar{a}_{k-1}$, where $\bar{a}_{i}$ is the binary complement of $a_{i}$ (that follows from $\left.2^{k}-1-f=\left(1-a_{0}\right)+2\left(1-a_{1}\right)+\cdots+2^{k-1}\left(1-a_{k-1}\right)=\bar{a}_{0}+2 \bar{a}_{1}+\cdots+2^{k-1} \bar{a}_{k-1}\right)$.

Lemma 8. Let $G=(V, E, w)\left(w: E \rightarrow \mathbb{Z}_{q}\right)$ be a weighted regular graph of parameters $\left(v ; r_{0}, r_{1}, \ldots, r_{q-1}\right)$. Then the complement $\bar{G}$ is a weighted regular graph of parameters $\left(v ; \bar{r}_{0}, \ldots, \bar{r}_{q-1}\right)$, where $\bar{r}_{q-1-j}=r_{j}$.

Proof. Let a be an arbitrary vertex. Recall that we denote by $N_{j}(\mathbf{a})$ the set of all neighbors of a vertex a of corresponding edge weight $j$. Since $G$ is weighted regular, then $\left|N_{j}(\mathbf{a})\right|=r_{j}$. In the graph $\bar{G}$, the weight $j$ will transform into $q-1-j$, therefore $\bar{r}_{q-1-j}=r_{j}$ and the lemma is shown.

Let $A \subset B$ and $x \in B$. As it is customary, we will denote by $x+A$ the set $\{x+a: a \in A\}$.

Theorem 9. Let $G=(V, E, w)\left(V \subseteq \mathbb{F}_{2}^{n}, w: E \rightarrow \mathbb{Z}_{q}\right)$ be an $(X ; Y)$-strongly regular, for some $X, Y \subseteq \mathbb{Z}_{q}$ with $|X|=2^{k-1}, q=2^{k}$, of parameters $\left(v ; r_{0}, r_{1}, \ldots, r_{q-1} ; e_{X}, d_{X}\right)$ such that $q-1-X=X$ or $\bar{X}$, and $q-1-Y=Y$. Then, the complement $\bar{G}$ is a $(q-1-X ; Y)$-strongly regular graph of parameters $\left(v ; \bar{r}_{0}, \ldots, \bar{r}_{q-1} ; \bar{e}_{q-1-X}, \bar{d}_{q-1-X}\right)$, where $\bar{r}_{q-1-j}=r_{j}, \bar{e}_{q-1-X}=e_{X}$ and $\bar{d}_{q-1-X}=d_{X}$, if $q-1-X=X$, respectively, $\bar{r}_{q-1-j}=r_{j}, \bar{e}_{q-1-X}=d_{X}$ and $\bar{d}_{q-1-X}=e_{X}$, if $q-1-X=\bar{X}$. 
Proof. The first claim follows from Lemma 8 . We consider the two cases $q-1-X=X$, or $\bar{X}$, separately. As before, for any two vertices $\mathbf{a}, \mathbf{b}$ we denote by $N_{Y}(\mathbf{a}, \mathbf{b})$ the set of all vertices $\mathbf{c}$ adjacent to both $\mathbf{a}, \mathbf{b}$ such that $w(\mathbf{a}, \mathbf{c}) \in Y, w(\mathbf{b}, \mathbf{c}) \in Y$.

Case 1. Let $q-1-X=X$. For any two vertices $\mathbf{a}, \mathbf{b}$ with $w(\mathbf{a}, \mathbf{b}) \in X$, then $\left|N_{Y}(\mathbf{a}, \mathbf{b})\right|=e_{X}$, since the weight of the edge between $\mathbf{a}, \mathbf{b}$ remains in $X$. Similarly, for two vertices $\mathbf{a}, \mathbf{b}$ with $w(\mathbf{a}, \mathbf{b}) \in \bar{X}$, then $\left|N_{Y}(\mathbf{a}, \mathbf{b})\right|=d_{X}$.

Case 2. Let $q-1-X=X$. For any two vertices $\mathbf{a}, \mathbf{b}$ with $w(\mathbf{a}, \mathbf{b}) \in X$, then the weight of the edge between $\mathbf{a}, \mathbf{b}$ in $\bar{G}$ is now in $\bar{X}$, and we know that in that case $N_{Y}(\mathbf{a}, \mathbf{b})=d_{X}$. Similarly, for two vertices $\mathbf{a}, \mathbf{b}$ with $w(\mathbf{a}, \mathbf{b}) \in \bar{X}$, then $\left|N_{Y}(\mathbf{a}, \mathbf{b})\right|=e_{X}$.

In the next theorem, we shall show a strong regularity theorem (a BernasconiCodenotti correspondence) for gbents $f \in \mathcal{G B}_{n}^{4}$ when $n$ even and $k=2$. For two vertices $\mathbf{a}, \mathbf{b}$ of the associated Cayley graph, for $i, j \in\{0,1,2,3\}$, let $N_{\{i, j\}}(\mathbf{a}, \mathbf{b})$ be the set of all "neighbor" vertices $\mathbf{w}$ to both $\mathbf{a}, \mathbf{b}$ such that the edges have additive weights $f(\mathbf{w} \oplus \mathbf{a}) \in\{i, j\}, f(\mathbf{w} \oplus \mathbf{b}) \in\{i, j\}$.

Theorem 10. Let $f \in \mathcal{G B}_{n}^{4}, n$ even. Then $f$ is gbent if and only if the associated generalized Cayley graph is $(X ; \bar{X})$-strongly regular with $e_{X}=d_{X}$, for both $X=\{0,1\}$, and $X=\{0,3\}$, that is, if and only if the following two conditions are satisfied:

(i) For any two pairs of vertices $\{\mathbf{a}, \mathbf{b}\},\{\mathbf{c}, \mathbf{d}\}$, then $\left|N_{\{2,3\}}(\mathbf{a}, \mathbf{b})\right|=\left|N_{\{2,3\}}(\mathbf{c}, \mathbf{d})\right|$.

(ii) For any two pairs of vertices $\{\mathbf{a}, \mathbf{b}\},\{\mathbf{c}, \mathbf{d}\}$, then $\left|N_{\{1,2\}}(\mathbf{a}, \mathbf{b})\right|=\left|N_{\{1,2\}}(\mathbf{c}, \mathbf{d})\right|$.

Proof. We know that $f=a_{0}+2 a_{1}$, where $a_{0}, a_{1} \in \mathcal{B}_{n}$, is gbent if and only if $a_{1}, a_{1} \oplus a_{0}$ are both bent (see [14, 15]). Let $\mathbf{u} \in \mathbb{V}_{n}$. We have that:

1. $f(\mathbf{u})=0 \Leftrightarrow a_{0}(\mathbf{u})=0,\left(a_{1} \oplus a_{0}\right)(\mathbf{u})=0$

2. $f(\mathbf{u})=1 \Leftrightarrow a_{0}(\mathbf{u})=1,\left(a_{1} \oplus a_{0}\right)(\mathbf{u})=1$

3. $f(\mathbf{u})=2 \Leftrightarrow a_{0}(\mathbf{u})=0,\left(a_{1} \oplus a_{0}\right)(\mathbf{u})=1$

4. $f(\mathbf{u})=3 \Leftrightarrow a_{0}(\mathbf{u})=1,\left(a_{1} \oplus a_{0}\right)(\mathbf{u})=1$

If $f$ is gbent, then $a_{1}, a_{1} \oplus a_{0}$ are both bent. Then, by [1], their respective graphs are srg with respective parameters $e=d, e^{\prime}=d^{\prime}$. We consider the following cases:

(a) Let any $\mathbf{a}, \mathbf{b}, \mathbf{c}$ such that $f(\mathbf{a} \oplus \mathbf{c}) \in\{1,2\}$ and $f(\mathbf{b} \oplus \mathbf{c}) \in\{1,2\}$, then $\left(a_{1} \oplus\right.$ $\left.a_{0}\right)(\mathbf{a} \oplus \mathbf{c})=1=\left(a_{1} \oplus a_{0}\right)(\mathbf{b} \oplus \mathbf{c})$. Since the graph corresponding to $a_{1} \oplus a_{0}$ is srg with $e^{\prime}=d^{\prime}$, then $\left|\left\{\mathbf{c}:\left(a_{1} \oplus a_{0}\right)(\mathbf{a} \oplus \mathbf{c})=1=\left(a_{1} \oplus a_{0}\right)(\mathbf{b} \oplus \mathbf{c})\right\}\right|=e^{\prime}$. Therefore, $\left|N_{\{1,2\}}(\mathbf{a}, \mathbf{b})\right|=e^{\prime}$.

(b) Let any $\mathbf{a}, \mathbf{b}$, $\mathbf{c}$ such that $f(\mathbf{a} \oplus \mathbf{c}) \in\{2,3\}$ and $f(\mathbf{b} \oplus \mathbf{c}) \in\{2,3\}$, then $a_{1}(\mathbf{a} \oplus \mathbf{c})=$ $1=a_{1}(\mathbf{b} \oplus \mathbf{c})$. Since the graph corresponding to $a_{1}$ is $\operatorname{srg}$ with $e=d$, then $\left|\left\{\mathbf{c}: a_{1}(\mathbf{a} \oplus \mathbf{c})=1=a_{1}(\mathbf{b} \oplus \mathbf{c})\right\}\right|=e$. Therefore, $\left|N_{\{2,3\}}(\mathbf{a}, \mathbf{b})\right|=e^{\prime}$. 
Conversely, let the generalized Cayley graph be such that, for any two pairs of vertices $\{\mathbf{a}, \mathbf{b}\},\{\mathbf{c}, \mathbf{d}\}$, then $\left|N_{\{2,3\}}(\mathbf{a}, \mathbf{b})\right|=\left|N_{\{2,3\}}(\mathbf{c}, \mathbf{d})\right|$, and $\left|N_{\{1,2\}}(\mathbf{a}, \mathbf{b})\right|=\left|N_{\{1,2\}}(\mathbf{c}, \mathbf{d})\right|$. As seen in the first part of the proof, $\left|N_{\{2,3\}}(\mathbf{a}, \mathbf{b})\right|=\left|\left\{\mathbf{c}: a_{1}(\mathbf{a} \oplus \mathbf{c})=1=a_{1}(\mathbf{b} \oplus \mathbf{c})\right\}\right|$. This number is a constant, regardless of the value of $a_{1}(\mathbf{a} \oplus \mathbf{b})$. This implies that the Cayley graph corresponding to $a_{1}$ is $\operatorname{srg}$ with $e=d$, where $e=\left|N_{\{2,3\}}(\mathbf{a}, \mathbf{b})\right|$.

Similarly, $\left|N_{\{1,2\}}(\mathbf{a}, \mathbf{b})\right|=\left|\left\{\mathbf{c}:\left(a_{1} \oplus a_{0}\right)(\mathbf{a} \oplus \mathbf{c})=1=\left(a_{1} \oplus a_{0}\right)(\mathbf{b} \oplus \mathbf{c})\right\}\right|$. This number is a constant, regardless of the value of $\left(a_{1} \oplus a_{0}\right)(\mathbf{a} \oplus \mathbf{b})$. This implies that the Cayley graph corresponding to $a_{1} \oplus a_{0}$ is $\operatorname{srg}$ with $e^{\prime}=d^{\prime}$, where $e^{\prime}=\left|N_{\{1,2\}}(\mathbf{a}, \mathbf{b})\right|$. Since both $a_{1}$ and $a_{1} \oplus a_{0}$ are therefore bent, we conclude that $f$ is gbent.

It is not hard to show that in some instances a "uniform" strong regularity will hold.

Corollary 11. Let $S$ be a bent set (see [3]), that is, every element of $S$ is a bent function and the sum of any two such is also a bent function. Let $a_{0}, a_{1} \in S$. Then, the generalized edge-weighted Cayley graph of $f=a_{0}+2 a_{1}$ is $(X ; \bar{X})$-strongly regular for any $X$ with $|X|=2$.

Remark 12. One certainly could inquire whether a similar result holds for a gbent for $n$ odd. Since the answer depends on a characterization (not currently known) of classical semibents in terms of their Cayley graphs, we leave that question for a subsequent project of an interested reader.

While we cannot find a necessary and sufficient condition on a gbent in $\mathcal{G B}_{n}^{q}, q=2^{k}$, we can follow a similar approach as in Theorem 10 to find a necessary condition on the Cayley graph of a generalized bent in $\mathcal{G B}_{n}^{q}$. As in the previous result, for $X \subseteq \mathbb{Z}_{q}$ and two vertices $\mathbf{u}, \mathbf{v}$, let $N_{X}(\mathbf{u}, \mathbf{v})$ be the set of vertices $\mathbf{w}$ such that $f(\mathbf{u} \oplus \mathbf{w}) \in X$ and $f(\mathbf{v} \oplus \mathbf{w}) \in X$. As usual, $\overline{\mathbf{c}}$ is the complement of the vector $\mathbf{c}$, and for two vectors $\mathbf{a}=$ $\left(a_{1}, \ldots, a_{t}\right), \mathbf{b}=\left(b_{1}, \ldots, b_{t}\right)$, the notation $\mathbf{a} \preceq \mathbf{b}$ means that $a_{i} \leq b_{i}$, for all $1 \leq i \leq t$. Recall that the canonical injection $\iota: \mathbb{V}_{s} \rightarrow \mathbb{Z}_{2^{s}}, \iota(\mathbf{c})=\mathbf{c} \cdot\left(1,2, \ldots, 2^{s-1}\right)=\sum_{j=0}^{s-1} c_{j} 2^{j}$, where $\mathbf{c}=\left(c_{0}, c_{1}, \ldots, c_{s-1}\right)$.

Theorem 13. Let $n$ be even, and $f=a_{0}+2 a_{1}+\cdots+2^{k-1} a_{k-1}, k \geq 2, a_{i} \in \mathcal{B}_{n}$, be a generalized Boolean function. If $f$ is gbent then the associated edge-weighted Cayley graph is $\left(X_{\mathbf{c}}^{0} ; X_{\mathbf{c}}^{1}\right)$-strongly regular with $e_{X_{\mathbf{c}}^{0}}=d_{X_{\mathbf{c}}^{0}}$, where $X_{\mathbf{c}}^{i}=\{\iota(\tilde{\mathbf{c}})+\iota(\mathbf{d}): \tilde{\mathbf{c}} \preceq$ $(\mathbf{c}, 1), w t(\tilde{\mathbf{c}}) \equiv i(\bmod 2), \mathbf{d} \preceq \overline{\mathbf{c}}\}, i=0,1$, for all $\mathbf{c} \in \mathbb{V}_{k-1}$; that is, for all $\mathbf{c} \in \mathbb{V}_{k-1}$, and for any two pairs of vertices $(\mathbf{u}, \mathbf{v}),(\mathbf{x}, \mathbf{y})$,

$$
\left|N_{X_{\mathbf{c}}^{1}}(\mathbf{u}, \mathbf{v})\right|=\left|N_{X_{\mathbf{c}}^{1}}(\mathbf{x}, \mathbf{y})\right| \text {. }
$$

Proof. The weighted regularity of $f$ follows from Proposition 6. If $f$ is gbent then by [10, Theorem 8], we know that for each $\mathbf{c} \in \mathbb{V}_{k-1}$, the Boolean function $f_{\mathbf{c}}$ defined as

$$
f_{\mathbf{c}}(\mathbf{x})=c_{0} a_{0}(\mathbf{x}) \oplus c_{1} a_{1}(\mathbf{x}) \oplus \cdots \oplus c_{k-2} a_{k-2}(\mathbf{x}) \oplus a_{k-1}(\mathbf{x})
$$

is a bent function with $\mathcal{W}_{f_{\mathbf{c}}}(\mathbf{a})=(-1)^{\mathbf{c} \cdot \iota^{-1}(g(\mathbf{a}))+s(\mathbf{a})} 2^{\frac{n}{2}}$, for some $g: \mathbb{V}_{n} \rightarrow \mathbb{Z}_{2^{k-1}}$, $s: \mathbb{V}_{n} \rightarrow \mathbb{F}_{2}$. 
While we cannot control in a simple manner the Walsh-Hadamard spectra conditions of $f_{\mathbf{c}}$ on the Cayley graph of a gbent $f$, we can derive some necessary conditions for $f$ to be gbent. Let $\mathbf{c} \in \mathbb{V}_{k-1}$ and $f_{\mathbf{c}}$ bent. Consider $\mathbf{u} \in \mathbb{V}_{n}$. Certainly, the condition that $f_{\mathbf{c}}(\mathbf{u})=1$ means that an odd number of functions $a_{j}$, occurring (that is, the corresponding coefficient is nonzero) in $f_{\mathbf{c}}$ will output 1 at $\mathbf{u}$. The $a_{j}$ 's corresponding to entries that are 0 in $\mathbf{c}$ can be taken either 0 or 1 (hence the condition in the definition of $X_{\mathbf{c}}^{i}$ that $\left.\mathbf{d} \preceq \overline{\mathbf{c}}\right)$. We see that the set of values of $f$ when $f_{\mathbf{c}}(\mathbf{u})=1$ is exactly $X_{\mathbf{c}}^{1}=\{\iota(\tilde{\mathbf{c}})+\iota(\mathbf{d}): \tilde{\mathbf{c}} \preceq(\mathbf{c}, 1), w t(\tilde{\mathbf{c}}) \equiv 1(\bmod 2), \mathbf{d} \preceq \overline{\mathbf{c}}\}$. Similarly, the set of values for $f$ when $f_{\mathbf{c}}(\mathbf{u})=0$ is $X_{\mathbf{c}}^{0}=\{\iota(\tilde{\mathbf{c}})+\iota(\mathbf{d}): \tilde{\mathbf{c}} \preceq(\mathbf{c}, 1), w t(\tilde{\mathbf{c}}) \equiv 0(\bmod 2), \mathbf{d} \preceq \overline{\mathbf{c}}\}$.

Since $f_{\mathbf{c}}$ is bent, then any two vertices, $\mathbf{u}, \mathbf{v}$, will have the same number of adjacent $\mathbf{w}$ with $f_{\mathbf{c}}(\mathbf{u} \oplus \mathbf{w})=f_{\mathbf{c}}(\mathbf{v} \oplus \mathbf{w})=1$, regardless of the value of $f_{\mathbf{c}}(\mathbf{u} \oplus \mathbf{v})$. This implies that $\left|N_{X_{\mathbf{c}}^{1}}(\mathbf{u}, \mathbf{v})\right|$ is constant for all $\mathbf{u}, \mathbf{v}$.

\section{$5 \quad$ Further comments}

We follow the notation of [7] and define yet another strong regularity concept here. Let $\Gamma$ be an edge-weighted graph (with no loops) with vertices $V$, edges $E$, and weight set $W$ (in [7], $W$ was taken to be $\mathbb{Z}_{q}^{*}$, although it could be arbitrary). As before, for each $\mathbf{u} \in V$ and $a \in W \cup\{0\}$, the weighted $a$-neighborhood of $u, N_{a}(\mathbf{u})$, is defined as follows:

- $N_{a}(\mathbf{u})=$ the set of all neighbors $\mathbf{v}$ of $\mathbf{u}$ in $\Gamma$ for which the edge $(\mathbf{u}, \mathbf{v}) \in E$ has weight $a$ (for each $a \in W$ ).

- $N^{0}(\mathbf{u})=$ the set of all nonadjacent $\mathbf{v}$ of $\mathbf{u}$ in $\Gamma$ (i.e., the set of $\mathbf{v}$ such that $(\mathbf{u}, \mathbf{v}) \notin E)$, that is, $N^{0}(\mathbf{u})=V \backslash \cup_{a \in W} N_{a}(\mathbf{u})$. In particular, $\mathbf{u} \in N^{0}(\mathbf{u})$.

In [7], the following definition of weighted strongly regular graph is given. Let $\Gamma$ be a connected edge-weighted graph which is regular as a simple (unweighted) graph. Let $W$ be the set of edge-weights of $\Gamma$. The graph $\Gamma$ is called an edge-weighted local strongly regular (to distinguish it from our definition we inserted the adjective "local") with parameters $v, k=\left(k_{a}\right)_{a \in W}, \lambda=\left(\lambda_{a}\right)_{a \in W^{3}}$, and $\mu=\left(\mu_{a}\right)_{a \in W^{2}}$, denoted $S R G_{W}(v, k, \lambda, \mu)$, if $\Gamma$ has $v$ vertices, and there are constants $k_{a}, \lambda_{a_{1}, a_{2}, a_{3}}$, and $\mu_{a_{1}, a_{2}}$, for $a, a_{1}, a_{2}, a_{3} \in W$, such that

$$
\left|N_{a}(\mathbf{u})\right|=k_{a} \text { for all vertices } \mathbf{u},
$$

and for vertices $\mathbf{u}_{1} \neq \mathbf{u}_{2}$ we have

$$
\left|N_{a_{1}}\left(\mathbf{u}_{1}\right) \cap N_{a_{2}}\left(\mathbf{u}_{2}\right)\right|= \begin{cases}\lambda_{a_{1}, a_{2}, a_{3}} & \text { if } \exists a_{3} \in W \text { with } \mathbf{u}_{1} \in N_{a_{3}}\left(\mathbf{u}_{2}\right) ; \\ \mu_{a_{1}, a_{2}} & \text { if } \mathbf{u}_{1} \notin N_{a_{3}}\left(\mathbf{u}_{2}\right) \text { for all } a_{3} .\end{cases}
$$

As was observed in [7] for functions $f: \mathbb{F}_{p^{n}} \rightarrow \mathbb{F}_{p}$, where several questions were posed, it is not clear what the connection between this concept and generalized (or $p$-ary) bentness is. Our strong regularity definition does allow us to show such a connection and in the case $k=2$, we have a complete Bernasconi-Codenotti correspondence [1, 2]. 


\section{References}

[1] A. Bernasconi, B. Codenotti, Spectral Analysis of Boolean Functions as a Graph Eigenvalue Problem, IEEE Trans. on Computers 48:3 (1999), 345-351.

[2] A. Bernasconi, B. Codenotti, J. M. VanderKam, A Characterization of Bent Functions in terms of Strongly Regular Graphs, IEEE Trans. on Computers, 50:9 (2001), 984-985.

[3] C. Bey, G. M. Kyureghyan, On Boolean functions with the sum of every two of them being bent, Des. Codes Cryptogr. 49 (2008), 341-346.

[4] N. Biggs, Algebraic Graph Theory (2nd ed.), Cambridge Univ. Press, 1993.

[5] L. Budaghyan, Construction and Analysis of Cryptographic Functions, SpringerVerlag, 2014.

[6] A. T. Butson, Generalized Hadamard matrices, Proc. Amer. Math. Soc. 13 (1962), 894-898.

[7] C. Celerier, D. Joyner, C. Melles, D. Phillips, S. Walsh, Edge-weighted Cayley Graphs and p-ary Bent Functions, Integers 16, A35, 2016.

[8] T. W. Cusick, P. Stănică, Cryptographic Boolean Functions and Applications, 2nd Ed. (Academic Press, San Diego, CA, 2017); 1st Ed., 2009.

[9] D. M. Cvetkovic, M. Doob, H. Sachs, Spectra of Graphs, Academic Press, 1979.

[10] T. Martinsen, W. Meidl, S. Mesnager, P. Stănică, Decomposing generalized bent and hyperbent functions, to appear in IEEE Trans. Inform. Th., 2017; DOI: 10.1109/TIT.2017.2754498.

[11] T. Martinsen, W. Meidl, P. Stănică, Generalized bent functions and their Gray images, In: Duquesne S., Petkova-Nikova S. (eds), Arithmetic of Finite Fields. WAIFI 2016, LNCS 10064. Springer, 160-173.

[12] T. Martinsen, W. Meidl, P. Stănică, Partial spread and vectorial generalized bent functions, Des. Codes Crypt. 85:1 (2017), 1-13.

[13] S. Mesnager, Bent functions: fundamentals and results, Springer Verlag, 2016.

[14] P. Solé, N. Tokareva, Connections between Quaternary and Binary Bent Functions, Prikl. Diskr. Mat. 1 (2009), 16-18 (see also, http://eprint.iacr.org/2009/544.pdf).

[15] P. Stănică, T. Martinsen, S. Gangopadhyay, B. K. Singh, Bent and generalized bent Boolean functions, Des. Codes Crypt. 69 (2013), 77-94.

[16] C. Tang, C. Xiang, Y. Qi, K. Feng, Complete characterization of generalized bent and $2^{k}$-bent Boolean functions, IEEE Trans. Inform. Th. 63:7 (2017), 4668-4674. 
[17] N. Tokareva, Generalizations of bent functions: a survey of publications, (Russian) Diskretn. Anal. Issled. Oper. 17 (2010), no. 1, 34-64; translation in J. Appl. Ind. Math. 5:1 (2011), 110-129.

[18] N. Tokareva, Bent Functions, Results and Applications to Cryptography, Academic Press, San Diego, CA, 2015. 